\title{
Real-time three-dimensional object reconstruction by use of a phase-encoded digital hologram
}

\author{
Osamu Matoba, Thomas J. Naughton, Yann Frauel, Nicolas Bertaux, and \\ Bahram Javidi
}

\begin{abstract}
A three-dimensional (3D) object reconstruction technique that uses only phase information of a phaseshifting digital hologram and a phase-only spatial-light modulator is proposed. It is well known that a digital hologram can store both amplitude and phase information of an optical electric field and can reconstruct the original $3 \mathrm{D}$ object in a computer. We demonstrate that it is possible to reconstruct optically $3 \mathrm{D}$ objects using only phase information of the optical field calculated from phase-shifting digital holograms. The use of phase-only information enables us to reduce the amount of data in the digital hologram and reconstruct optically the $3 \mathrm{D}$ objects using a liquid-crystal spatial light modulator without optical power loss. Numerical evaluation of the reconstructed 3D object is presented. (C) 2002 Optical Society of America

OCIS codes: $200.4560,100.6890,090.1760,100.3010$.
\end{abstract}

\section{Introduction}

Three-dimensional (3D) imaging, display, and processing have been investigated frequently. ${ }^{1-5}$ Holography is one popular technique that can record and reconstruct $3 \mathrm{D}$ objects. Recently, digital holography $^{6}$ has become viable with the ongoing development of megapixel CCD sensors that have sufficient dynamic range in each pixel. It has been shown with numerical propagation that the fully complex field calculated from the digital holograms in the Fresnel domain can be used to reconstruct 3D objects successfully. ${ }^{6-9}$ Storage of the hologram in a computer enables us to reduce the noise through imageprocessing techniques and numerically reconstruct

O. Matoba is with the Institute of Industrial Science, University of Tokyo, 4-6-1, Komaba, Meguro-ku, Tokyo 153-8505 Japan. T. J. Naughton is with the Department of Computer Science, National University of Ireland, Maynooth, County Kildare, Ireland. Y. Frauel and B. Javidi are with the Department of Electrical and Computer Engineering, University of Connecticut, U-157, 260 Glenbrook Road, Storrs, Connecticut 06269-2157. N. Bertaux is with Domaine Universitaire de Saint Jérôme, Ecole Nationale Superieure de Physique de Marseille, 13397 Marseille, Cedex 20, France.

Received 27 December 2001; revised manuscript received 7 June 2002.

0003-6935/02/296187-06\$15.00/0

(C) 2002 Optical Society of America the object with arbitrary views. The digital hologram is also in a convenient form for data transmission and object recognition. ${ }^{10,11}$ With digital reconstruction, however, it takes a long time to calculate the Fresnel diffraction pattern. This makes it difficult to develop a real-time $3 \mathrm{D}$ display device.

In this paper, we propose a real-time $3 \mathrm{D}$ object reconstruction system that uses phase-only information of the complex field from the digital holograms and a phase-only spatial light modulator. It is well known that the phase distribution, that is a wave front, contains 3D position information of point sources. Phase-only hologram or information is used in kinoform ${ }^{12,13}$ and diffractive optical elements for interconnection, ${ }^{14,15}$ imaging or optical elements such as lenses and prisms. The proposed method is suitable for real-time optical reconstruction of the 3D object because phase-only reconstruction allows us to use commercially available display devices, such as liquid-crystal spatial light modulator(s) (SLM)(s).16,17 These devices can only be operated in either phase or amplitude modulation mode. Using only this phase information, we can reduce by half the storage requirements of the digital hologram. We also take advantage of minimal optical power loss in the reconstruction process. Reconstructed 3D objects with phase-only information are evaluated numerically. An experimental demonstration with a liquid-crystal spatial light modulator is also presented. 


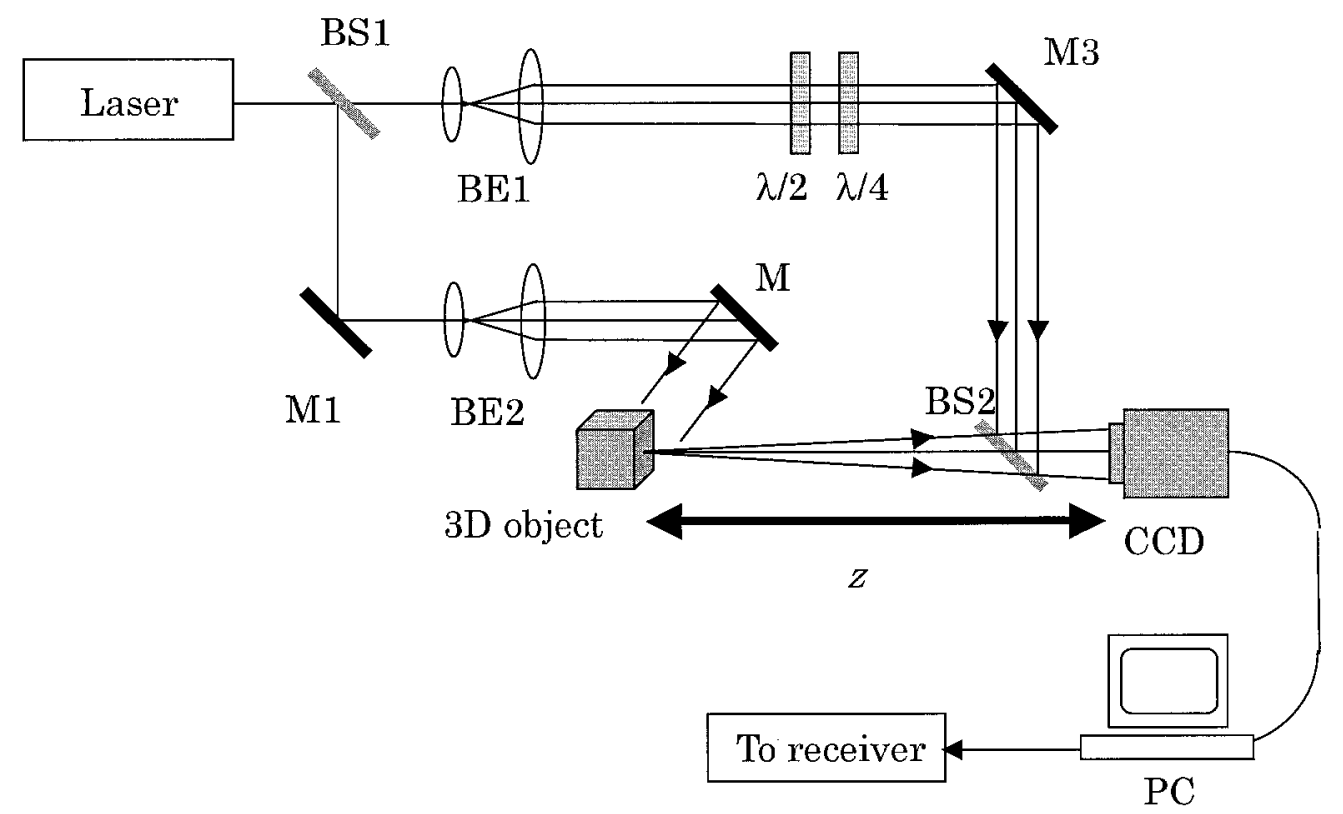

(a)

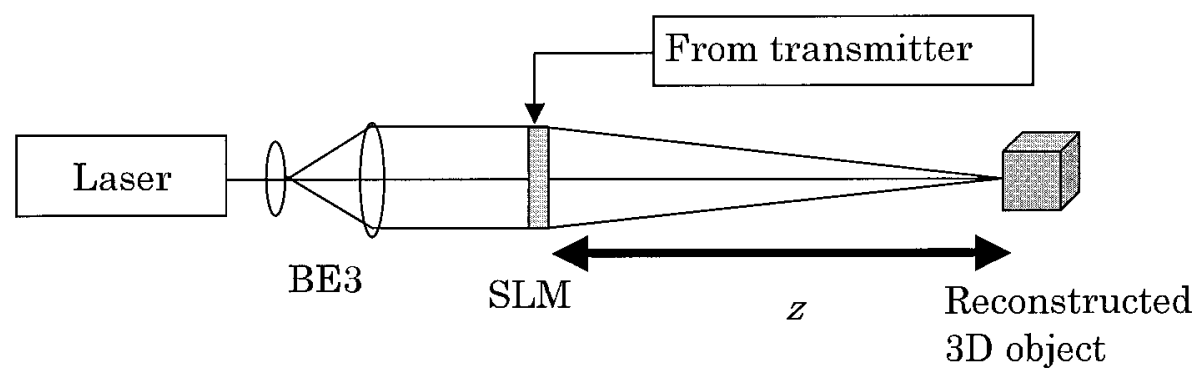

(b)

Fig. 1. Proposed 3D object recording and reconstruction system. (a) Recording system for digital holograms by use of a phase-shifting interferometer, (b) optical reconstruction system by use of a phase-only information of digital holograms.

\section{Reconstruction with Phase-Only Information}

An illustration of the proposed 3D object recording and reconstruction system is presented in Fig. 1. The proposed system consists of two subsystems: One is a recording system for digital holograms of $3 \mathrm{D}$ objects and the other is an optical reconstruction system by use of phase-only data of a digital hologram.

For the recording system, we use a Mach-Zehnder interferometer architecture as a phase-shifting interferometer. A laser beam is divided into two beams by beamsplitter BS1. A 3D object is illuminated by a collimated laser beam and diffracts this beam. Because 3D objects used in experiments are small enough compared with the CCD size and the object is located far enough from the CCD, we consider the paraxial region where the scalar diffraction theory is valid. The diffraction pattern propagates to a CCD camera that is located at a distance $z$ along the optical axis and is described by using the Fresnel approximation as

$$
\begin{aligned}
\tilde{U}\left(x^{\prime}, y^{\prime}\right)= & A\left(x^{\prime}, y^{\prime}\right) \exp \left\{j \phi\left(x^{\prime}, y^{\prime}\right)\right\} \\
= & \frac{1}{j \lambda} \iiint U(x, y, z) \frac{1}{z} \exp \{j k z\} \\
& \times \exp \left\{\frac { j k } { 2 z } \left\{\left(x^{\prime}-x\right)^{2}\right.\right. \\
& \left.\left.+\left(y^{\prime}-y\right)^{2}\right\}\right\} \mathrm{d} x \mathrm{~d} y \mathrm{~d} z
\end{aligned}
$$

where $\lambda$ and $k$ are the wavelength and the wavenumber of the illumination, respectively. In Eq. (1), $A\left(x^{\prime}, y^{\prime}\right)$ and $\phi\left(x^{\prime}, y^{\prime}\right)$ are, respectively, the amplitude and the phase of the complex optical electric field at 
the CCD plane. The diffracted beam interferes in line with a reflected plane wave (reference beam) by use of beamsplitter BS2. To reconstruct the complex field of the 3D object as described in Eq. (1) from the digital holograms, we employ a phase-shifting digital holography technique. ${ }^{7}$ This technique is effective to remove the conjugate reconstruction even when an in-line hologram is used. To implement phase-shifting digital holography, the reference beam passes through both a half-wave plate and a quarterwave plate as shown in Fig. 1(a). Phase retardations in the reference beam of $0, \pi / 2, \pi$, and $3 \pi / 2$ can be achieved by controlling the positions of the fast and slow axes of the two plates. By use of the resulting four digital interferograms, the complex field of the 3D object in Eq. (1) can be calculated as described in Refs. 7 and 8.

It has already been shown that with computer reconstruction the fully complex field of the 3D object calculated from the digital holograms can be successfully reconstructed. Using the complex field of the hologram, we can reconstruct numerically the $3 \mathrm{D}$ object by the Fresnel-Kirchhoff integral, written by

$$
\begin{aligned}
R(x, y ; z)= & -\frac{1}{j \lambda} \iint \tilde{U}\left(x^{\prime}, y^{\prime}\right) \frac{1}{z} \exp \{-j k z\} \\
& \times \exp \left\{-\frac{j k}{2 z}\left\{\left(x-x^{\prime}\right)^{2}\right.\right. \\
& \left.\left.+\left(y-y^{\prime}\right)^{2}\right\}\right\} \mathrm{d} x^{\prime} \mathrm{d} y^{\prime} .
\end{aligned}
$$

In the proposed method that uses phase-only information, a reconstructed object is described as

$$
\begin{aligned}
P(x, y ; z)= & -\frac{a}{j \lambda} \iint \exp \left\{j \phi\left(x^{\prime}, y^{\prime}\right)\right\} \frac{1}{z} \exp \{-j k z\} \\
& \times \exp \left\{-\frac{j k}{2 z}\left\{\left(x-x^{\prime}\right)^{2}\right.\right. \\
& \left.\left.+\left(y-y^{\prime}\right)^{2}\right\}\right\} \mathrm{d} x^{\prime} \mathrm{d} y^{\prime}
\end{aligned}
$$

where

$$
a=\left[\frac{\iint|R(x, y ; z)|^{2} \mathrm{~d} x \mathrm{~d} y}{\iint|P(x, y ; z)|^{2} \mathrm{~d} x \mathrm{~d} y}\right]^{1 / 2} .
$$

Note that the total powers of Eqs. (2) and (3) have been made equivalent by choosing $a$ as indicated in Eq. (4). In the next section we evaluate the quality of the reconstructed $3 \mathrm{D}$ object using phase-only information.

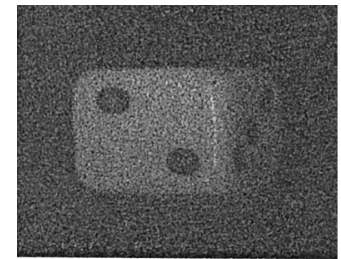

(a)

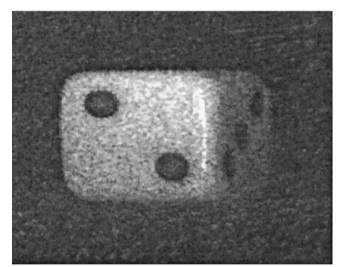

(c)

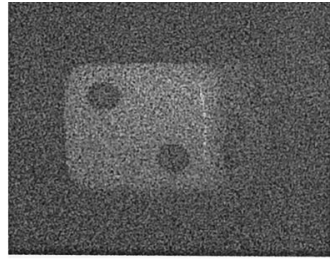

(b)

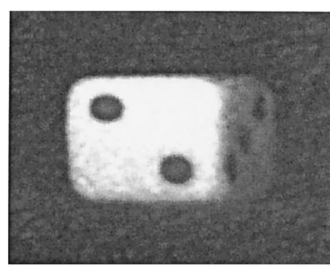

(d)
Fig. 2. Numerically reconstructed 3D objects of die from (a) a fully complex field, (b) phase-only information, (c) low-pass filtered $3 \mathrm{D}$ objects with a mean filter of $11 \times 11$ pixels, (d) $21 \times 21$ pixels after reconstruction with phase-only information.

\section{Numerical Evaluation}

To evaluate the error in the reconstructed 3D object, we calculate a normalized root mean square (NRMS) criterion as follows:

$$
\begin{aligned}
& \text { NRMS } \\
& =\frac{\left[\iint\left\{|R(x, y ; z)|^{2}-|P(x, y ; z)|^{2}\right\}^{2} \mathrm{~d} x \mathrm{~d} y\right]^{1 / 2}}{\left[\iint\left\{|R(x, y ; z)|^{2}\right\}^{2} \mathrm{~d} x \mathrm{~d} y\right]^{1 / 2}} .
\end{aligned}
$$

Note that the total powers in Eqs. (2) and (3) can be made equivalent by adjusting $a$ in Eq. (4).

Digital holograms of the 3D object are recorded optically as shown in Fig. 1(a). Numerically reconstructed 3D objects are evaluated. An argon ion laser operated at a wavelength of $514.5 \mathrm{~nm}$ is used as a recording beam. The CCD array consists of $2028 \times$ 2044 pixels. Each pixel is $9 \mu \mathrm{m} \times 9 \mu \mathrm{m}$ and has 1024 gray levels (10 bits). We use two $3 \mathrm{D}$ objects in the experiments: One is a die with dimensions 5 $\mathrm{mm} \times 5 \mathrm{~mm} \times 5 \mathrm{~mm}$, and the other is a screw of similar size. The die and the screw are located at distances of $322 \mathrm{~mm}$ and $390 \mathrm{~mm}$ from the CCD, respectively.

Figures 2(a) and 2(b) show the numerically reconstructed 3D die object by use of complex information and phase-only information, respectively. Figures $3(\mathrm{a})$ and $3(\mathrm{~b})$ show the numerically reconstructed $3 \mathrm{D}$ screw object by use of complex information and phase-only information, respectively. These figures show that the phase-only information can reconstruct the 3D object successfully. We can also see that speckle noise is an influence on both $3 \mathrm{D}$ 


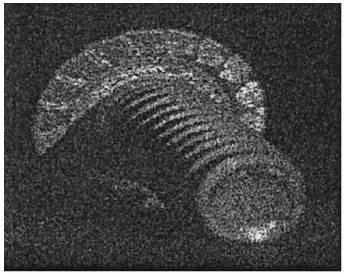

(a)

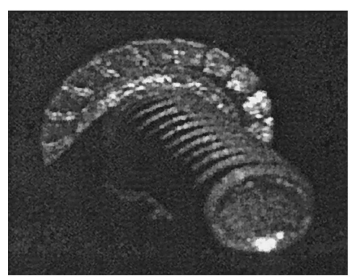

(c)

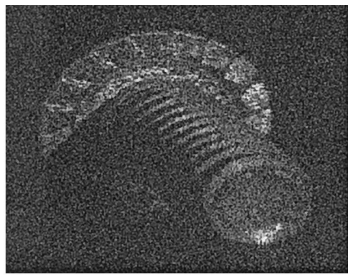

(b)

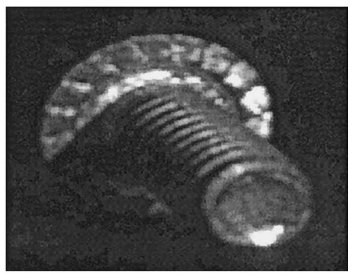

(d)
Fig. 3. Numerically reconstructed 3D objects of a screw from (a) a fully complex field, (b) phase-only information, (c) low-pass filtered 3D objects with a mean filter of $11 \times 11$ pixels, (d) $21 \times 21$ pixels after reconstruction with phase-only information.

reconstructed images. The speckle is a key in this method. It is important to mention that the speckle on the hologram is necessary if we want to reconstruct the object with the phase-only information. The effect of the speckle is to spread the intensity histogram of the hologram, so that the equalization error of the amplitude is reduced.

We evaluate the reconstructed $3 \mathrm{D}$ object. The reconstructed 3D objects are low-pass filtered by the mean filter to reduce the speckle noise in the reconstructed image. The low-pass filtered images are presented in Figs. 2(c), 2(d), 3(c), and 3(d). The sizes of the mean filter in Figs. 2(c) and 3(c) are $11 \times 11$ pixels, and in Figs. $2(\mathrm{~d})$ and $3(\mathrm{~d})$ are $21 \times 21$ pixels. The intensity levels are scaled to enhance the contrast. We can see that speckle noise is removed to a large extent by low-pass filtering. The intensities of the die and the screw reconstructed from the phaseonly holograms are compared with the intensities of the objects reconstructed from fully complex holograms, for different mean filtering neighborhoods. Figure 4 shows a NRMS difference as a function of the side length of the mean filter. We can see that the NRMS difference is reduced as the size of the mean filter increases for both $3 \mathrm{D}$ objects. In the die $3 \mathrm{D}$ object, the error is reduced from $40 \%$ to $15 \%$.

Here we discuss the effect of the mean filter and the remaining error in Fig. 4. The mean filter is used to remove the multiplicative speckle noise. Ideally the filter would give the mean of the original object multiplied by the mean of the noise, which is a constant all over the image. Unfortunately, the size of the mean filter that is $N \times N$ is finite. When $N$ is finite we can only obtain an estimate of the mean of the noise. This estimate has a standard deviation that decreases when $N$ increases. This standard devia-

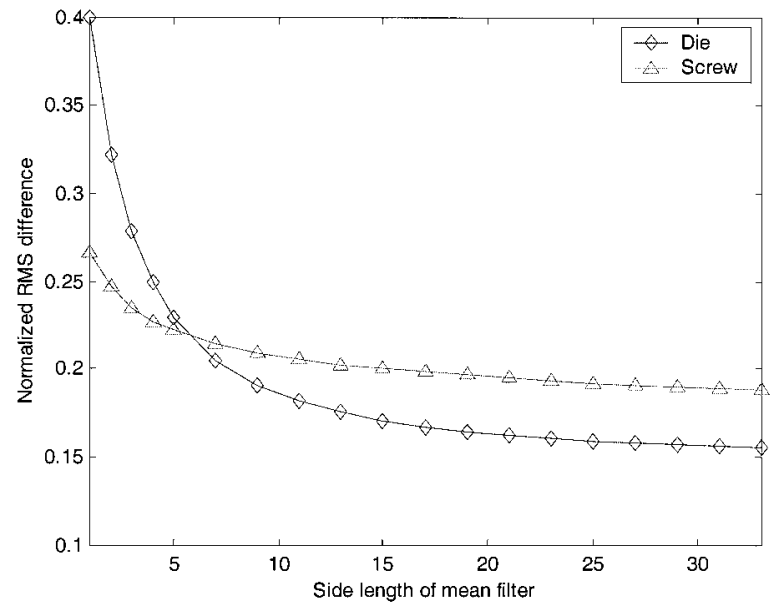

Fig. 4. NRMS difference between reconstructed 3D objects from a fully complex field and reconstructions from phase-only information as a function of the side length of the mean filter.

tion is a noise that limits the accuracy that we can expect when we compare two reconstructed images from the phase-only hologram and the fully complex hologram. For a small value of $N$, this accuracy is low, which is responsible most of the RMS error. When $N$ increases, the RMS error decreases until it is only due to the actual difference between the images. The remaining error is due to the loss of amplitude information of the hologram. We expect that these errors can be decreased by manipulating the phase distribution by use of any error reduction technique, such as simulated annealing or genetic algorithm. ${ }^{18}$

In practical systems, there is a limit on the number of phase levels that can be displayed in phaseonly spatial light modulators. Die and screw reconstructions from phase-only information are compared with intensity images reconstructed from fully complex information, for different numbers of quantization levels. For these calculations, we use a mean filter with $11 \times 11$ pixels. Figures 5 and 6 show the reconstructed 3D objects without quantization reduction, and with quantization reductions to 4 bits and 1 bit. We can discern the $3 \mathrm{D}$ objects even when the phase level of the phase-only spatial light modulators is binary. Figure 7 shows NRMS as a function of the number of phase levels of phaseonly information. As few as 16 levels (4 bits) are sufficient to reconstruct the $3 \mathrm{D}$ objects without significant loss of detail.

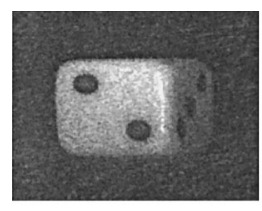

(a)

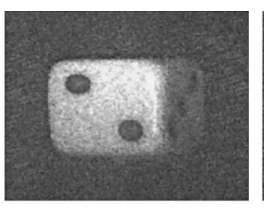

(b)

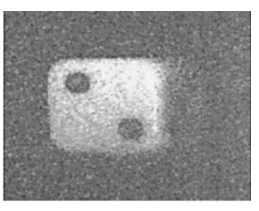

(c)
Fig. 5. Reconstructed 3D object of the die without quantization reduction and with quantization reductions to 4 bits and 1 bit. 


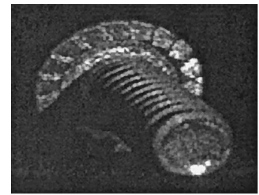

(a)

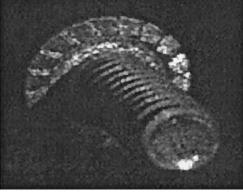

(b) (c)

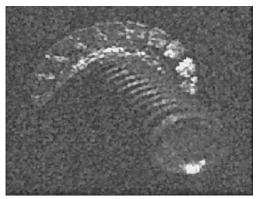

Fig. 6. Reconstructed 3D object of the screw without quantization reduction and with quantization reductions to 4 bits and 1 bit.

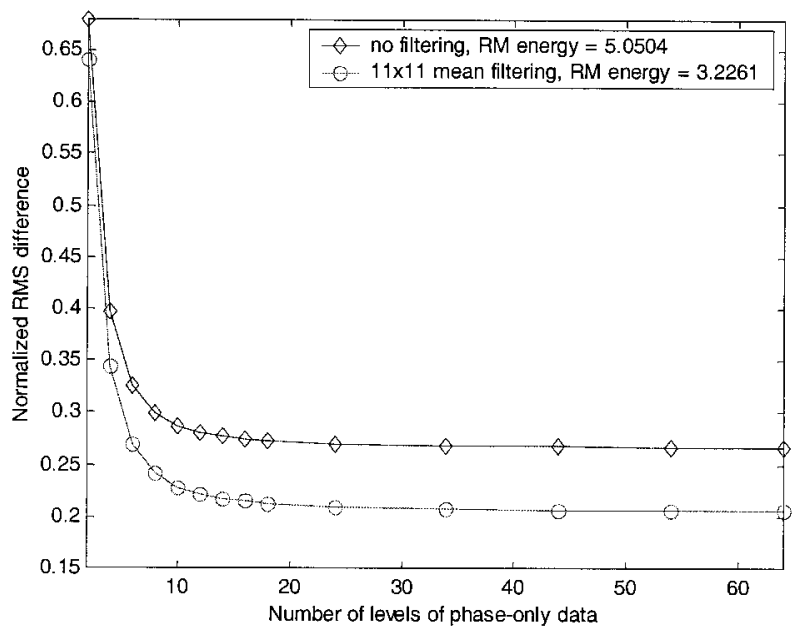

(a)

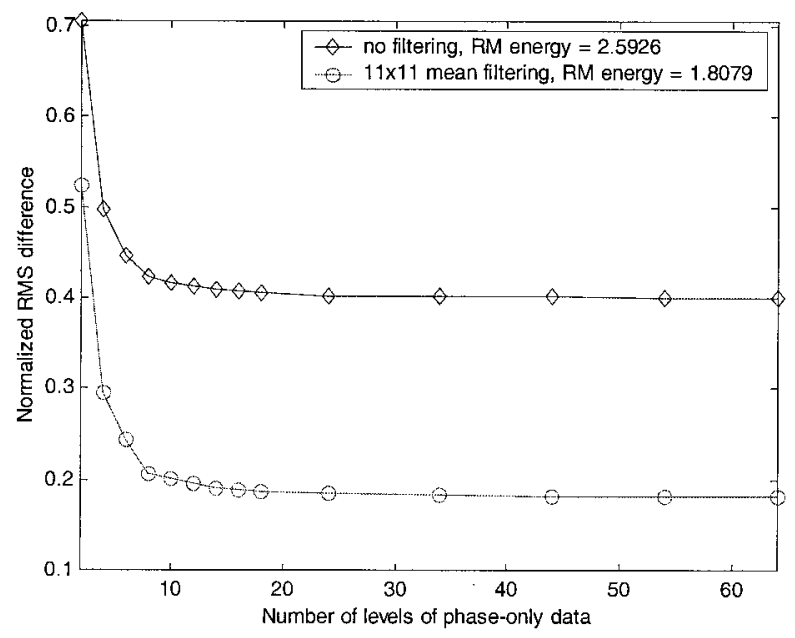

(b)

Fig. 7. NRMS difference as a function of the number of phase levels of phase-only information when 3D objects are (a) a screw and (b) a die, respectively.

\section{Optical Reconstruction}

The optical setup to reconstruct 3D objects is shown in Fig. 1(b). A He-Ne laser operated at a wavelength of $632.8 \mathrm{~nm}$ is used. After the collimation and the beam-width expansion of the He-Ne laser

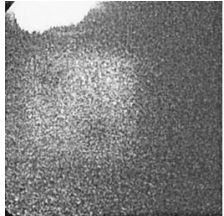

(a)

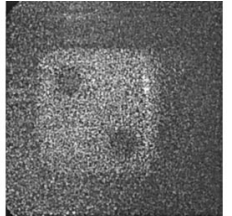

(b)

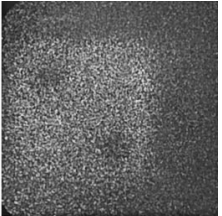

(c)
Fig. 8. Experimental results: (a), (b), and (c) are reconstructed images of the die where the CCD is located at $113 \mathrm{~mm}, 123 \mathrm{~mm}$, and $133 \mathrm{~mm}$ from the SLM, respectively.

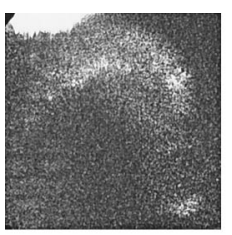

(a)

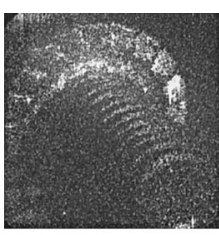

(b)

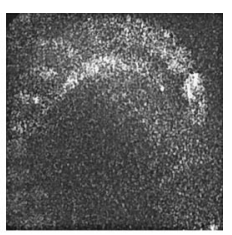

(c)
Fig. 9. Experimental results: (a), (b), and (c) are reconstructed images of the screw where the CCD is located at $135 \mathrm{~mm}, 155 \mathrm{~mm}$, and $165 \mathrm{~mm}$ from the SLM, respectively.

beam, the beam illuminates a liquid-crystal SLM with $1024 \times 768$ pixels. The size of each pixel is approximately $18 \mu \mathrm{m} \times 18 \mu \mathrm{m}$. This SLM does not have enough pixels to display the complete $2044 \times$ 2028 pixel phase-only hologram, and so a $1024 \times$ 768 pixel window is presented. By measuring phase retardation of the SLM in a Mach-Zehnder interferometer, the maximum amount of phase retardation possible with our SLM is $0.6 \pi$. The phase retardation is almost linearly proportional to the signal level from the computer. Figures 8 and 9 show the reconstructed $3 \mathrm{D}$ die and screw objects. Figures 8(a), 8(b), and 8(c) contain reconstructions with the CCD located at distances of $113 \mathrm{~mm}, 123 \mathrm{~mm}$, and $133 \mathrm{~mm}$ from the SLM, respectively. Figures 9(a), 9(b) and 9(c) show reconstructions at distances of $135 \mathrm{~mm}, 155 \mathrm{~mm}$, and $165 \mathrm{~mm}$ from the SLM, respectively. Figures 8(b) and 9(b) are the most in focus. From these figures, we can see that the die and the screw are reconstructed at different planes. The calculated positions of the in-focus reconstructions are $104 \mathrm{~mm}$ and $126 \mathrm{~mm}$, respectively. These errors are caused by a small amount of phase retardation of the SLM, our use of a different readout wavelength, different pixel size, and a readout wavefront not exactly the same as a plane wave.

\section{Conclusions}

We have proposed a method to reconstruct $3 \mathrm{D}$ objects that uses only the phase information of the complex field from digital holograms. Experimental demonstration shows that optical reconstruction of views of $3 \mathrm{D}$ objects can be successfully implemented. $\mathrm{Nu}-$ merical evaluation shows that NRMS errors of from $10 \%$ to $40 \%$ appear due to speckle noise. These errors were caused by the loss of amplitude information 
of the hologram. The error could be decreased by manipulation of the hologram's phase distribution. We think that the proposed method is a promising way for a real-time 3D display because the phaseonly reconstruction allows us to use commercially available display devices such as liquid-crystal SLMs.

\section{References}

1. J. W. Goodman, Introduction to Fourier Optics, 2nd ed. (McGraw-Hill, New York, 1996).

2. H. J. Caulfield, Handbook of Optical Holography (Academic, New York, 1979).

3. F. Okano, J. Arai, H. Hoshino, and I. Yuyama, "Threedimensional video system based on integral photography," Opt. Eng. 38, 1072-1077 (1999).

4. A. D. McAulay, Optical Computer Architectures: The Application of Optical Concepts to the Next Generation of Computers (Wiley, New York, 1991).

5. N. Yoshikawa and T. Yatagai, "Fringe pattern correlator for three-dimensional object recognition," Opt. Lett. 25, 14241426 (2000).

6. Y. Li, K. Itoh, W. Watanabe, K. Yamada, D. Kuroda, J. Nishii, Y. Jiang, "Three-dimensional hole drilling of silica glass from the rear surface with femtosecond laser pulses," Opt. Lett. 26, 1912-1914 (2001).

7. U. Schnars and W. Jüptner, "Direct recording of holograms by a CCD target and numerical reconstruction," Appl. Opt. 33, 179-181 (1994).

8. I. Yamaguchi and T. Zhang, "Phase-shifting digital holography," Opt. Lett. 22, 1268-1270 (1997).

9. B. Javidi and E. Tajahuerce, "Three-dimensional object recog- nition by use of digital holography," Opt. Lett. 25, 610-612 (2000).

10. M. Sutkowski and M. Kujawinska, "Application of liquid crystal (LC) devices for optoelectronic reconstruction of digitally stored holograms," Opt. Lasers Eng. 33, 191-201 (2000).

11. E. Tajahuerce, O. Matoba, and B. Javidi, "Shift-invariant three-dimensional object recognition by means of digital holography," Appl. Opt. 40, 3877-3886 (2001).

12. Y. Frauel, E. Tajahuerce, M. A. Castro, and B. Javidi, "Distortion-tolerant three-dimensional object recognition with digital holography," Appl. Opt. 40, 3887-3893 (2001).

13. J. A. Jordan, Jr., P. M. Hirsch, L. B. Lesem, and D. L. Van Rooy, "Kinoform lenses," Appl. Opt. 9, 1883-1887 (1970).

14. T. H. Barnes, T. Eiju, K. Matuda, H. Ichikawa, M. R. Taghizadeh, and J. Turunen, "Reconfigurable free-space optical interconnections with a phase-only liquid crystal spatial light modulator," Appl. Opt. 31, 5527-5535 (1992).

15. K. L. Tan, S. T. Warr, I. G. Manolis, T. D. Wilkinson, M. M. Redmond, W. A. Crossland, R. J. Mears, and B. Robertson, "Dynamic holography for optical interconnections. II. Routing holograms with predictable location and intensity of each diffraction order," J. Opt. Soc. Am. A 18, 205-215 (2001).

16. L. G. Neto, D. Roberge, and Y. Sheng, "Programmable optical phase-mostly holograms with coupled-mode modulation liquid-crystal television,” Appl. Opt. 34, 1944-1950 (1995).

17. Y. Igasaki, F. Li, N. Yoshida, H. Toyoda, T. Inoue, N. Mukohzaka, Y. Kobayashi, and T. Hara, "High efficiency electricallyaddressable phase-only spatial light modulator," Opt. Rev. 6, 339-344 (1999).

18. N. Yoshikawa and T. Yatagai, "Phase optimization of a kinoform by simulated annealing," Appl. Opt. 33, 863-868 (1994). 